\title{
Core Collapse in Rotating Massive Stars and LGRBs
}

\author{
Aldo Batta \\ Instituto de Astronomía, Universidad Nacional Autónoma de México, Apdo. postal 70-264 \\ Ciudad Universitaria, D.F., México \\ email: abatta@astro.unam.mx
}

\begin{abstract}
The collapse of massive rotating stellar cores and the associated accretion is thought to power long GRBs. The physical scale and dynamics of the accretion disk are initially set by the angular momentum distribution in the progenitor, and the physical conditions make neutrino emission the main cooling agent in the flow. We have carried out an initial set of calculations of the collapse of rotating polytropic cores in three dimensions, making use of a pseudo-relativistic potential and a simplified cooling prescription. We focus on the effects of self gravity and cooling on the overall morphology and evolution of the flow for a given rotation rate in the context of the collapsar model. For the typical cooling times expected in such a scenario we observe the appearance of strong instabilities on a time scale, $t_{\text {cool }}$, following disk formation. Such instabilities and their gravitational interaction with the black hole $(\mathrm{BH})$ produce significant variability in the energy loss and accretion rates, which would translate into neutrino cooling variations when a more realistic neutrino cooling scheme is implemented in future work.
\end{abstract}

Keywords. Accretion disks, instabilities, hydrodynamics

\section{Introduction}

From GRB afterglow observations of the optical and $X$-ray band, it has been possible to locate the origin of these sources at cosmological distances. They also show that long GRBs can sometimes be associated with a core collapse SN (with no H lines, i.e. type Ib or Ic) taking place at the same time and at the same place. A review by Woosley \& Bloom (2006) shows the existing evidence for the link of long GRB at low redshift with type Ic SNe, and the progenitor mechanisms currently explored.

In this work we will study the collapsar model proposed by Woosley (1993), to explain the formation of a GRB from a pre-supernova (PreSN) star whose shutdown of nuclear reactions at the central region induces the collapse of the core, and later on of the whole star. Eventually, the core will collapse into a $\mathrm{BH}$ that would be able to accrete the remaining infalling material from the star. With the high temperatures and densities the material reaches at the disk, neutrino emmision becomes the main cooling mechanism. These neutrinos may provide the energy to power the GRB, with the obtained neutrino luminosities $\left(L_{\nu}\right)$ from the collapsar, contributing to the production of a relativistic jet through neutrino annihilation.

Important works have been carried out in 2D and 3D (e.g. MacFadyen \& Woosley 1999, and Taylor et al. 2010, respectively) for this collapsar model, but there is not a complete study on the importance of structure formation in the accretion flow and or heating/cooling mechanisms. This work focuses on the study of the effects of self gravity and cooling on $3 \mathrm{D}$ simulations of the collapse of a rotating polytropic envelope onto a $\mathrm{BH}$ in the context of the collapsar model. More specific details on the initial conditions and the input physics are given in the next section. 

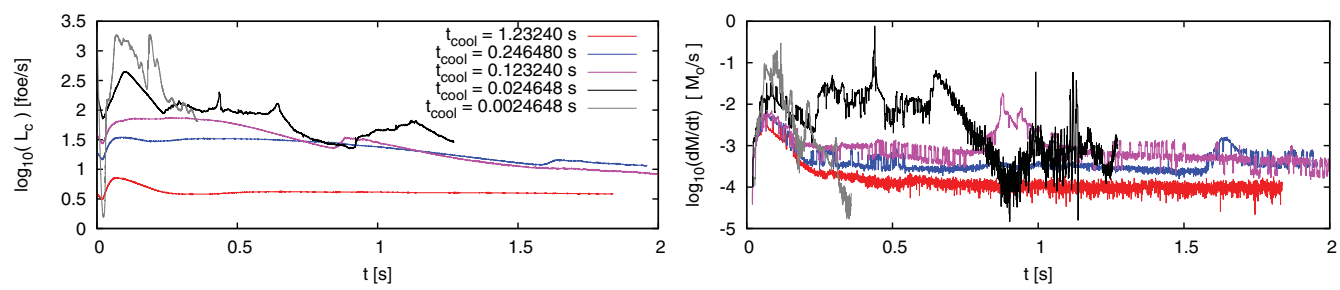

Figure 1. Logarithm of the energy loss rate $L_{c}$ (left panel) in foes for models with $t_{\text {cool }}=1.23 \mathrm{~s}$ (red line), $t_{\text {cool }}=0.24 \mathrm{~s}$ (blue line), $t_{\text {cool }}=0.12 \mathrm{~s}$ (pink line), $t_{\text {cool }}=0.02 \mathrm{~s}$ (orange line) and $t_{\text {cool }}=0.002 \mathrm{~s}$ (gray line) and logarithm of the $\mathrm{BH}$ accretion mass rate $\dot{M}$ in solar masses per second (right panel).

\section{Overview}

For the densities and temperatures expected near the $\mathrm{BH}$, one can estimate the neutrino cooling time scale in the gas. By considering an approximate EOS (internal energy u) composed by relativistic non-degenerated electron-positron pairs and an ideal gas, and a neutrino cooling prescription $q_{\nu}$ (Narayan et al. 2001), one can estimate the neutrino cooling time scale as follows:

$$
t_{\nu}=u / q_{\nu} \quad \text { where } u=\frac{3}{2} \frac{k T \rho}{\mu m_{p}}+\frac{11}{4} a T^{4}, q_{\nu} \simeq 5 \times 10^{33} T_{11}^{9}+9.0 \times 10^{23} \rho T_{11}^{6}
$$

This turns into a neutrino cooling time scale ranging from a few seconds to $\sim 10^{-4} \mathrm{~s}$ for $\rho=10^{11}$ to $10^{12} \mathrm{~g} \mathrm{~cm}^{-3}$ and $T=10^{10}$ to $10^{11} \mathrm{~K}$.

With this $t_{\nu}$ timescale in mind, we study the importance of the neutrino cooling efficiency by means of a simplified cooling prescription with a characteristic cooling time $\left(t_{\text {cool }}\right)$. Each fluid element is cooled according to

$$
\frac{d u}{d t}=-\frac{u}{t_{c o o l}}
$$

where $t_{\text {cool }}=1.23,0.24,0.12,0.024$, and $0.002 \mathrm{~s}$ are the cooling times explored in our simulations. For a fixed rigid body rotation rate (just below breakup) we studied the differences obtained by using each of these cooling times on the collapse and accretion of $2.5 M_{\odot}$ polytropic envelopes onto a $2 M_{\odot} \mathrm{BH}$. These envelopes where constructed by solving the Lane-Emdem hydrostatic equilibrium equation for a $\gamma=5 / 3$ and $4.5 M_{\odot}$ polytropic star with a radius of $R_{s} \simeq 1700 \mathrm{~km}$. All simulations were made using a personal version of the code GADGET-2 (Springel et al. 2001, Springel 2005).

Mass Accretion and Energy Loss Rates. By looking at the accretion and energy loss rates ( $\dot{M}$ and $L_{c}$ respectively) showed in Fig. 1 we can see that increasing cooling efficiency translates into more profuse and more intense variations in both $L_{c}$ and $\dot{M}$. These strong variations seem to occur at the same time on both quantities, which implies they must be produced by the same phenomena. Given that by construction, strong variations on $L_{c}$ are only possible if there is an increase in the internal energy $u$ of the gas, there must be a contraction or shock at the disk material producing such increase in order to obtain intense variations in $L_{c}$. By looking after structure formation at the disk, we will be able to see if it is responsible for the intense variations on both $L_{c}$ and $\dot{M}$.

Cooling Efficiency $\&$ Structure Formation. In order to obtain information about the formation of non-axisymmetric instabilities, we performed a Fourier transform of the 

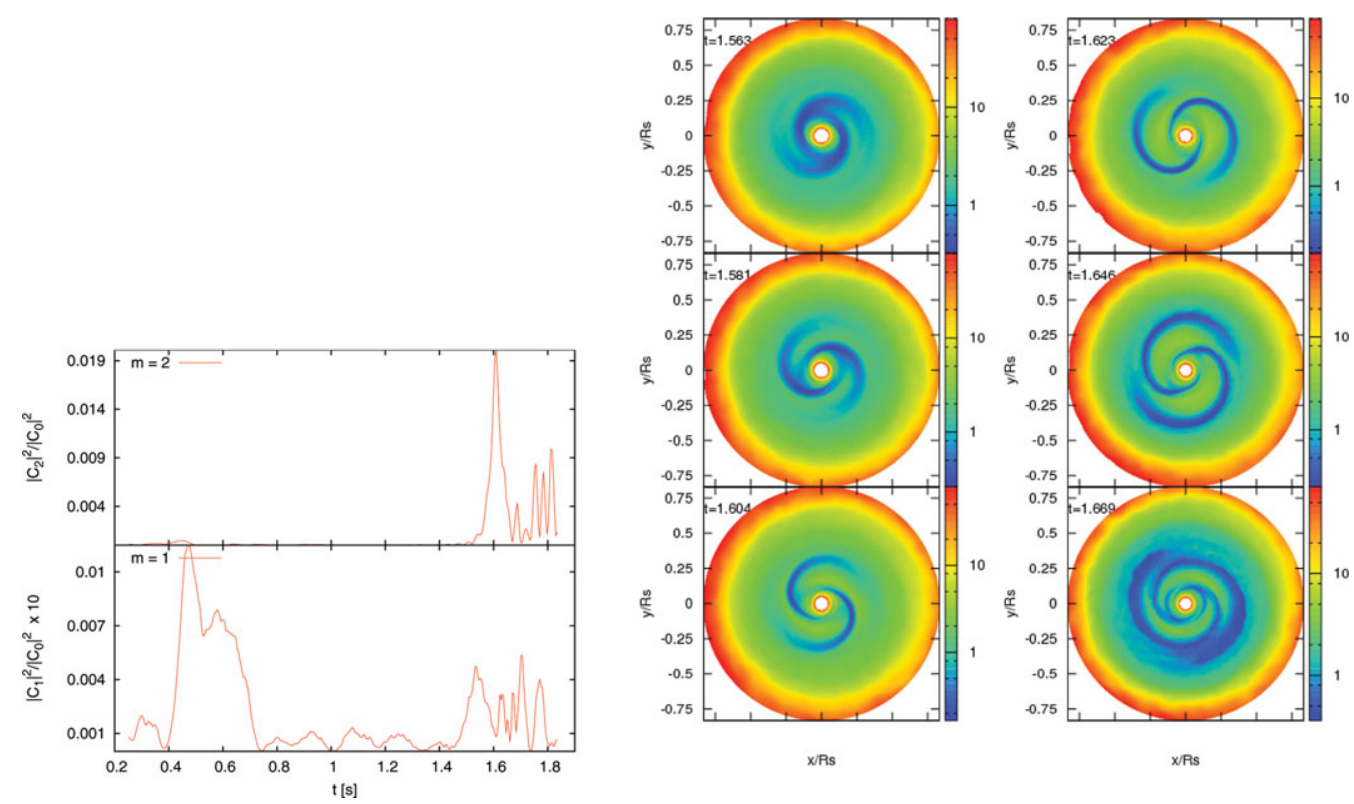

Figure 2. Relative power $\left|c_{m}\right|^{2}=\left|C_{m}\right|^{2} /\left|C_{0}\right|^{2}(m=1,2,3,4)$ of the azimuthal distribution of mass $\left(\Phi_{M}\right)$ Fourier transform of the model with $t_{c o o l}=0.24 \mathrm{~s}$ (left panel) and evolution of the Toomre parameter $Q$ at times $\simeq 1.6 \mathrm{~s}$ (right panel). Deep blue regions have $Q<1$. There is noticeable spiral structure forming at the same time strong variations on $L_{c}$ and $\dot{M}$ appear.

azimuthal distribution of mass $\Phi_{M}=\int\left[\int \rho(\phi, r, z) d z\right] r d r$ (as in Zurek \& Benz 1986) defining the amplitude of the mode $m$ by

$$
C_{m}=\frac{1}{2 \pi} \int_{0}^{2 \pi} e^{i m \phi} \Phi_{M} d \phi
$$

The relative power $\left|c_{m}\right|^{2}=\left|C_{m}\right|^{2} /\left|C_{0}\right|^{2}$ indicates the intensity of $m$ spiral arms compared to the disk integrated mass. If such spiral structures are present in the disk, they should be visible in density or internal energy maps; moreover, they should also be visible as unstable regions by plotting the Toomre parameter $Q_{T}=\kappa c_{s} /(\pi G \Sigma)$, determined by the superficial density $\Sigma$, the local sound speed $c_{s}$ and the epicyclic frequency $\kappa$.

Figure 2 shows the relative power $\left|c_{m}\right|^{2}$ as a function of time (left panel) and the Toomre parameter evolution near $t=1.6 \mathrm{~s}$ (right panel) for the model with $t_{\text {cool }}=0.24$ s. As shown in Fig 1, the strong variation on $L_{c}$ and $\dot{M}$ is caused by the formation of two spiral arms shown as an intense increase in $\left|c_{2}\right|^{2}$ at $t \simeq 1.6 \mathrm{~s}$. Further increase of the cooling efficiency induces formation of gas clumps due to the collapse of highly unstable regions. These can be seen on Fig. 3, showing the relative power $\left|c_{m}\right|^{2}$ as a function of time (left panel) and the Toomre parameter evolution near $t=0.4 \mathrm{~s}$ (right panel) for the model with $t_{c o o l}=0.024 \mathrm{~s}$. Intense structure formation episodes shown in the left panel, coincide in time with intense variations shown in both $L_{c}$ and $\dot{M}$. Some of these variations are considerably narrower and are due to the close encounter of a gas clump with the $\mathrm{BH}$, which produces an intense spiral arm of material falling onto the $\mathrm{BH}$. 

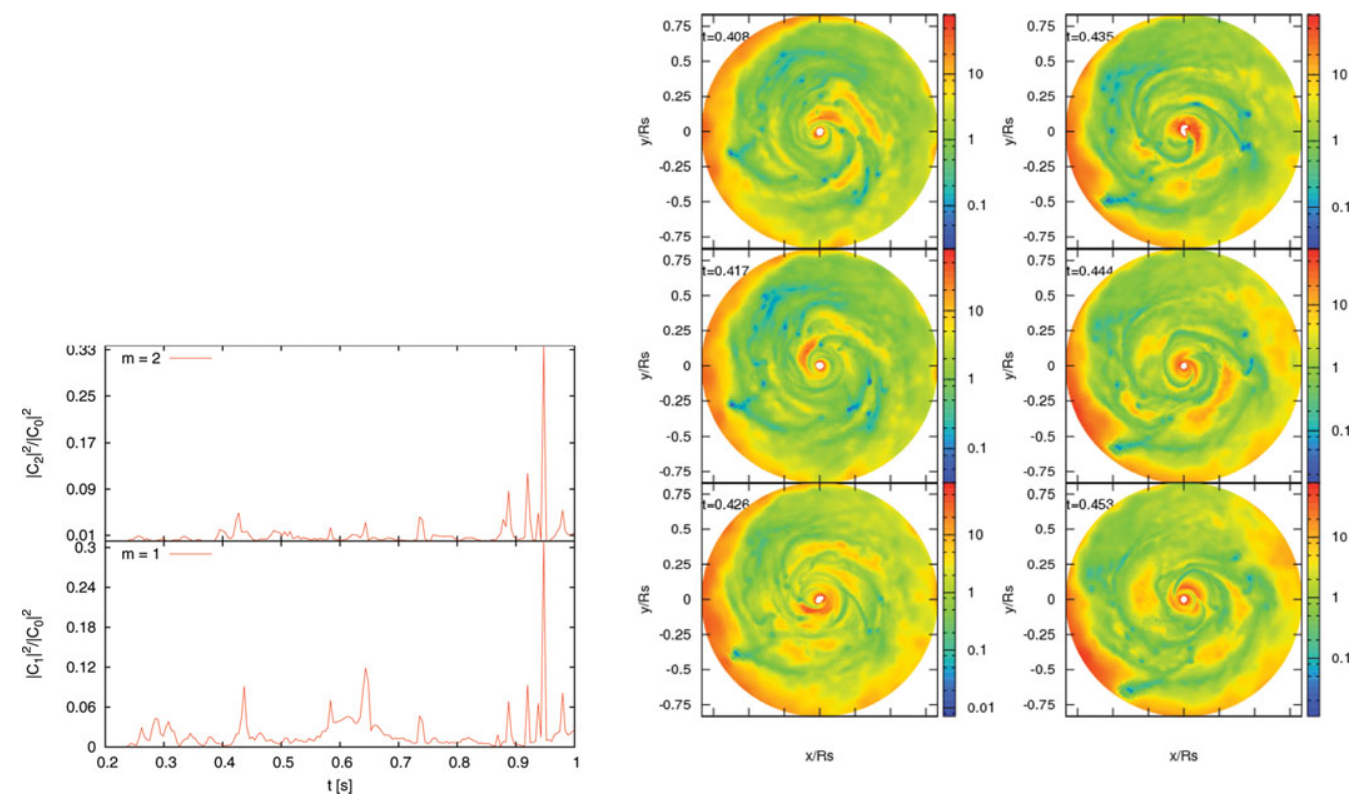

Figure 3. Relative power $\left|c_{m}\right|^{2}=\left|C_{m}\right|^{2} /\left|C_{0}\right|^{2} \quad(m=1,2,3,4)$ for the azimuthal's distribution of mass $\left(\Phi_{M}\right)$ Fourier transform of the model with $t_{c o o l}=0.024 \mathrm{~s}$ (left panel) and evolution of the Toomre parameter $Q$ at times $\simeq 0.4 \mathrm{~s}$ (right panel). Deep blue regions have $Q<0.1$. There is clump formation since early times and noticeable spiral structure forming at the same time strong variations on $L_{c}$ and $\dot{M}$ appear.

\section{Implications}

As shown by our simulations, if neutrino cooling is efficient enough to induce the formation of structure at the accretion disk, the energy loss and accretion rates would have intense variations whose duration depends on the time those structures remain. Such intense and localized variations could change significantly the neutrino energy deposition on the infalling layers that have not reached the disk yet. Neutrino cooling efficiency (and therefore, structure formation) will depend on the initial rotation rate of the progenitor (Taylor et al. 2010). In the case of really collapsed and massive structures (such as gas clumps and intense spiral arms), the intrinsic symmetry break in the gravitational interaction with the $\mathrm{BH}$ could be intense enough to considerably move the $\mathrm{BH}$ from its original position. Allowing the $\mathrm{BH}$ to move could change its gravitational interaction with intense structures, and therefore, the accretion and energy loss rates obtained from the disk. Further work will explore such scenario.

\section{References}

MacFadyen, A. I. \& Woosley, S. E. 1999, ApJ 524, 262

Narayan, R., Piran, T., \& Kumar, P. 2001, ApJ, 557, 949

Springel, V., Yoshida, N., \& White, S. D. M. 2001, New Astronomy, 6, 51

Springel, V. 2005, MNRAS, 364, 1105

Taylor, P. A., Miller, J. C., \& Podsiadlowski, P. 2010, arxiv:1006.4624v1 


\section{Discussion}

MetzGer: What about the ultimate fate of the clumps? Do you expect to have any runaway cooling?

BATTA: Clumps that are not accreted by the $\mathrm{BH}$ or stay in orbits around the $\mathrm{BH}$ get scattered to outer regions, even though they may not get to far away given that they have to interact with the rest of the gas at the disk and the infalling material. Ultimately if the clump becomes very dense, cooling will be less efficient due to increased optical depth to neutrinos. 